\title{
Processos erosivos e produção de sedimentos: suas relações com a cobertura vegetal na Bacia do Ribeirão do Roque
}

\author{
Erosive processes and sediment yield: its relations with \\ vegetation cover in Roque River Basin
}

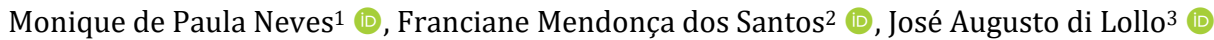

${ }^{1}$ Universidade Federal de São Carlos - UFSCar, São Carlos, SP, Brasil. E-mail: moniqueneves.ambiental@gmail.com ${ }^{2}$ Faculdade de Ciências, Universidade de Lisboa, Lisboa, Portugal. E-mail: fran.mendonca@hotmail.com

3Universidade Estadual Paulista "Júlio de Mesquita Filho" - Unesp, Ilha Solteira, SP, Brasil. E-mail: jose.lollo@unesp.br
\end{abstract}

\begin{abstract}
Como citar: Neves, M. P., Santos, F. M., \& di Lollo, J. A. (2021). Processos erosivos e produção de sedimentos: suas relações com a cobertura vegetal na Bacia do Ribeirão do Roque. Revista de Gestão de Água da América Latina, 18, ê17. https://doi.org/10.21168/rega.v18e17
\end{abstract}

RESUMO: Este estudo tem como objetivo analisar a ocorrência de processos erosivos e a produção de sedimentos, e sua relação com a cobertura vegetal na Bacia do Ribeirão do Roque - SP. Por meio do índice topográfico de capacidade de transporte de sedimento (ITCTS) constatou-se que os locais com maior potencial de ocorrência de erosão e deposição localizam-se próximo das cabeceiras de drenagem e nos terrenos mais íngremes. 0 índice de vegetação por diferenças normalizada (NDVI, em inglês) para o ano 2002 indica que a vegetação fornece proteção contra os processos erosivos nas áreas identificadas como susceptíveis aos processos, o que é comprovado pelo menor número de processos erosivos na área quando comparado com o ano de 2010 e 2018. As declividades médias e os vales foram os principais contribuintes topográficos para a ocorrência dos processos erosivos potencializados pela remoção da vegetação principalmente para os anos de 2010 e 2018. Análises da água no exutório da bacia mostram maior concentração de sedimentos, indicando aumento da produção de sedimentos entre os anos de 2008 e 2011, fato relacionado ao aumento do número de processos erosivos, que por sua vez podem estar associados com o aumento das áreas agrícolas, e ausência de práticas de manejo do solo.

Palavras-chave: Processos Erosivos; ITCTS; NDVI; Produção de Sedimentos.

ABSTRACT: This study aims to analyze the occurrence of erosive processes and sediment yield, and their relationship with vegetation cover in the Roque River Basin - SP. Through the topographic sediment transport capacity (TCI) it was found that the areas with the highest potential for erosion and deposition are located near the drainage headwaters and the steepest terrain and showed compliance with the points identified in the study area. The Normalized Differential Vegetation Index (NDVI) for the year 2002 indicates that vegetation provides protection against erosion in areas identified as susceptible, as evidenced by the lower number of erosion processes in the area when compared to 2010 and 2018. Average slopes and valleys were the main topographic contributors to the occurrence of erosive processes enhanced by vegetation removal mainly for the years 2010 and 2018. Analysis of water show the increase of sediment yield between the 2008 and 2011, related to the increasing of erosive processes in the area. These processes may be associated with the increase of arable land in the region and inadequate land use practices.

Keywords: Erosive Processes; TCI; NVDI; Sediment Yield.

\section{INTRODUÇÃO}

A erosão do solo causada pela ação da água é considerada um dos tipos mais graves de erosão e atraiu considerável atenção no passado, principalmente devido aos seus efeitos destrutivos, incluindo eutrofização, poluição não pontual e, eventualmente, degradação do solo.

Recebido: Maio 19, 2021. Revisado: Agosto 11, 2021. Aceito: Setembro 22, 2021. 
Especificamente nos países tropicais, a erosão ocorre devido aos índices pluviométricos elevados, que podem concentrar-se em determinadas épocas do ano, agravando ainda mais o processo (Guerra, 1998; Asselman et al., 2003; Singer \& Shainberg, 2004; Jin et al., 2008; Vaz et al., 2021).

Tanto os processos erosivos quanto a produção de sedimentos são indicadores importantes que podem ser aplicados ao controle de qualidade das águas superficiais. A produção de sedimentos é a quantidade de solo removida para os rios e lagos em um determinado período em uma área definida, e é um processo importante na erosão do solo das bacias hidrográficas (Ouyang et al., 2010).

Um dos índices utilizados na modelagem da erosão é o Índice Topográfico de Capacidade de Transporte de Sedimento (ITCTS) que representa o efeito na topografia na erosão, e auxilia na predição das áreas de erosão e de deposição. 0 índice foi formulado considerando a relação entre o fator topográfico, definido pela multiplicação do fator de comprimento de rampa (L) e o fator de declividade (S), e a produção de sedimento por unidade de área propostos por Wischmeier \& Smith (1978). Com base nesses conceitos e na teoria de potência de fluxo unitário, Moore \& Burch (1986) propôs o ITCTS para representar o efeito dos processos hidrológicos nos processos erosivos.

Segundo Minella \& Merten (2012), a utilização desses índices é uma importante ferramenta de planejamento ambiental, pois favorece não só a identificação de áreas suscetíveis à erosão, como também a tomada de decisão na implantação de práticas de manejo e conservação do solo.

No entanto, a cobertura vegetal é um dos fatores mais importantes que oferecem proteção do solo contra a erosão e sua variação é altamente dependente do manejo da terra. Usando dados de satélite, existem diferentes maneiras de lidar com a dinâmica espacial e temporal da vegetação. Índices de vegetação como o NDVI (índice de vegetação de diferença normalizada) pode ser facilmente derivado de dados adquiridos por uma variedade de satélites operando em diferentes resoluções espaciais e temporais (Vrieling et al., 2008).

De acordo com Carvalho et al. (2014), o NDVI é um dos principais índices utilizados no monitoramento da vegetação, o que permite o monitoramento das mudanças espaciais e temporais da superfície. A partir da avaliação deste índice é possível inferir sobre a influência da vegetação nos processos erosivos principalmente em áreas com características do meio físico que favoreçam os processos.

O índice de vegetação com diferença normalizada (NDVI) é calculado a partir de dados de sensoriamento remoto multiespectral e permite identificar a presença de vegetação, corpos d'água, áreas abertas, áreas de matagal, áreas montanhosas, áreas agrícolas, florestas densas e demais coberturas do solo (Gandhi et al., 2015).

Nesse contexto, esse trabalho teve como objetivo utilizar o Índice Topográfico de Capacidade de Transporte de Sedimento (ITCTS) e o Índice de Vegetação por Diferenças Normalizadas (NDVI) na Bacia Hidrográfica do Ribeirão do Roque (SP) para avaliar a ocorrência de processos erosivos. A área de estudo apresenta como predominante o uso da terra agrícola, o que favorece o surgimento de processos erosivos quando associado às características intrínsecas do meio e justifica a elaboração do estudo realizado.

\section{CARACTERIZAÇÃO DA ÁREA DE ESTUDO}

A área de estudo compreende a Bacia Hidrográfica do Ribeirão do Roque (BHRR) localizada na Unidade de Gerenciamento de Recursos Hídricos 9 (UGRHI-9) no médio Mogi-Guaçu. A área é limitada pelas coordenadas UTM 230000-265000 E e 753000 - 7565000 N, zona 23S e possui área de aproximadamente $458 \mathrm{~km}^{2}$ (Figura 1).

Quanto à geologia a Bacia do Ribeirão do Roque localiza-se na Bacia Sedimentar do Paraná (Figura 2), na qual ocorrem com maior predominância folhelhos variegados da Formação Corumbataí, arenitos fluviais da Formação Pirambóia e intrusivas básicas da Formação Serra Geral. As demais unidades correspondem a depósitos recentes do Quaternário (IG, 2017). É importante destacar que os materiais associados à Formação Pirambóia $(41,70 \%)$ são muito arenosos e, portanto, altamente susceptíveis a erosão. 

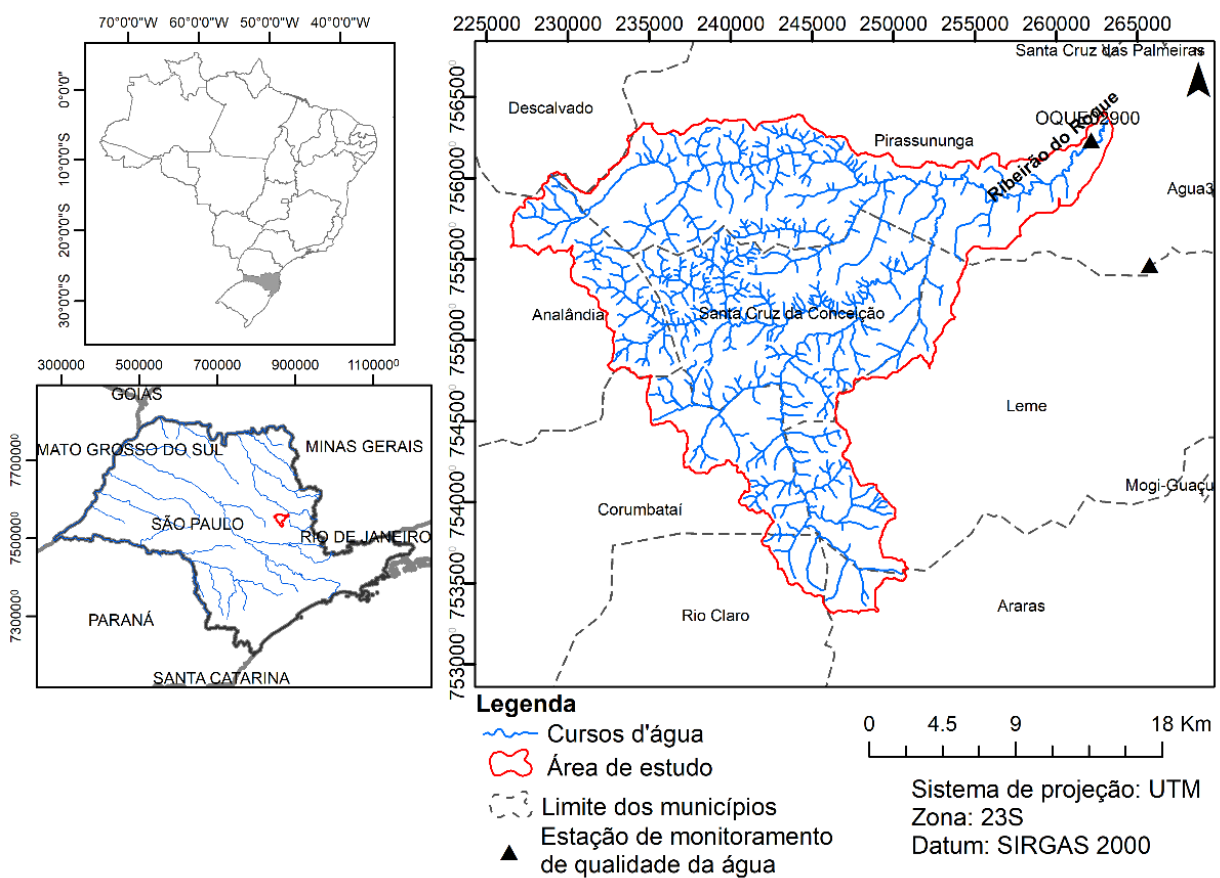

Figura 1 - Localização da área de estudo (Bacia Hidrográfica do Ribeirão do Roque - BHRR) e estação de monitoramento da qualidade da água. Fonte: Autores (2021)

Considerando a classificação de unidades morfoesculturais de Ross \& Moroz (1997) a área de estudo é composta por quatro unidades: Planalto Residual de São Carlos, Depressão Mogi-Guaçu e Morro Isolado. Essas unidades são constituídas, principalmente por colinas com topos aplanados, vales pouco entalhados com baixa densidade de drenagem.

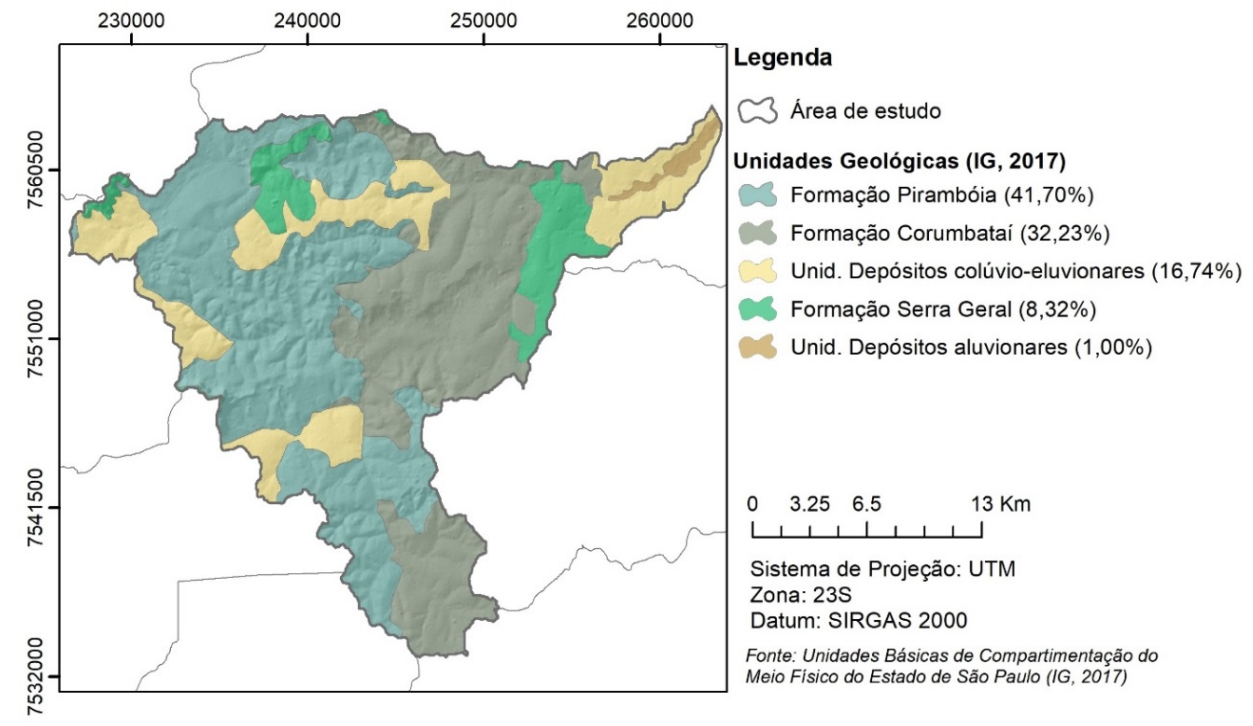

Figura 2 - Mapa de unidades geológicas da Bacia Hidrográfica do Ribeirão do Roque (BHRR). Fonte: Autores (2021)

O clima predominante na área em estudo, segundo a classificação de Köppen é o Cwa, clima tropical de altitude com verão chuvoso e inverno seco (Alvares et al., 2013). A temperatura e a precipitação média anual são respectivamente $22^{\circ} \mathrm{C}$ e $1200 \mathrm{~mm}$.

Segundo Anjinho et al., (2018), o Rio São Roque é um dos principais mananciais de abastecimento regional do Médio Mogi-Guaçu. 


\section{MATERIAIS E MÉTODOS}

Para o desenvolvimento deste estudo foram utilizados os seguintes dados: Modelo Digital de Elevação - MDE - ALOS - PALSAR, com resolução espacial de 12,5 m (Japan Aerospace Exploration Agency, 2019); imagens Landsat 2002, 2010 e 2018 com resolução espacial de 30 metros (Instituto Nacional de Pesquisas Espaciais, 2018); Agência Nacional de Águas (ANA) e softwares ArcGIS 10.5 e Google Earth Pro. A Figura 3 apresenta um fluxograma simplificado com o método de trabalho adotado.

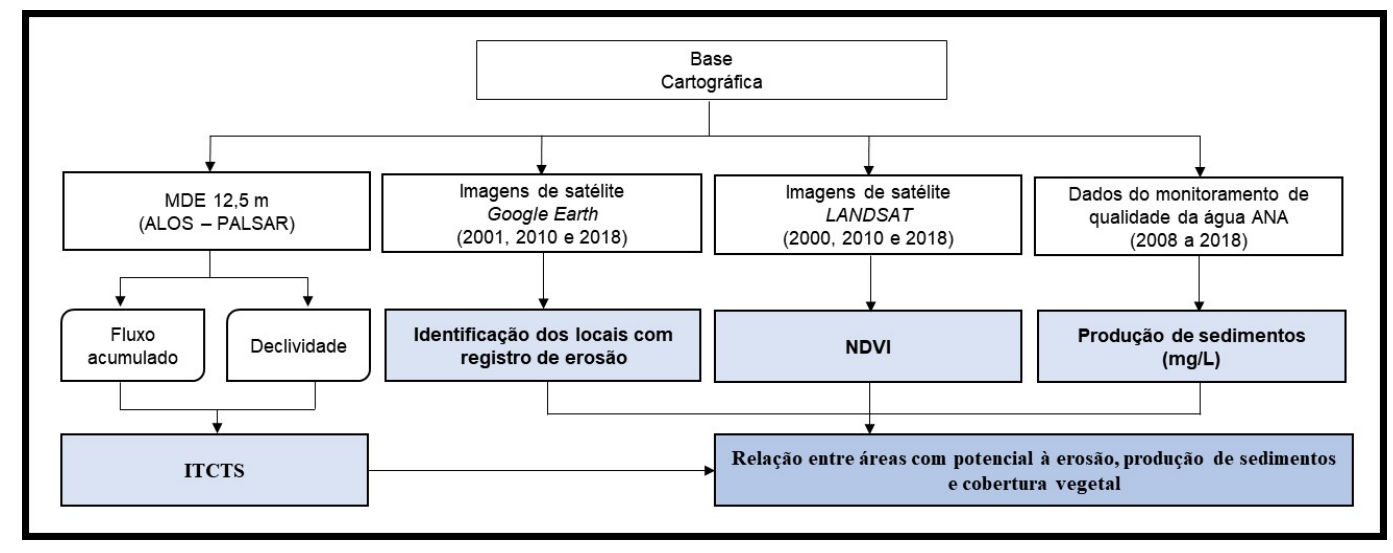

Figura 3 - Fluxograma com as etapas de trabalho.

\section{ÍNDICE TOPOGRÁFICO DE CAPACIDADE DE TRANSPORTE DE SEDIMENTOS}

0 Índice Topográfico de Capacidade de Transporte de Sedimentos (ITCTS) representa as áreas com potencial de ocorrência de erosão e deposição (Moore \& Burch, 1986; Wilson \& Gallant, 1996). O ITCTS representa a distribuição espacial da capacidade de transporte de sedimento, pixel a pixel, com base na área de contribuição específica (As) e na declividade $(\tan \beta)$ segundo a Equação 1.

ITCTS $=L S=\left(\frac{A_{S}}{a_{0}}\right)^{m}\left(\frac{\tan _{\beta}}{b_{0}}\right)^{n}$

Onde: As é a área de contribuição específica $\left(\mathrm{m}^{2} / \mathrm{m}\right) ; \tan \beta$ é a declividade (graus); $a_{0}$ e $b_{0}$ são equivalentes ao fator de comprimento e inclinação; e $m$ e $n$ são constantes, sendo $\mathrm{m}=1,3 \mathrm{e}$ $\mathrm{n}=0,6$.

De acordo com Moore e Burch (1986), os valores de $a_{0}$ e $b_{0}$, são $22,13 \mathrm{~m}$ e $0,0896 \mathrm{~m} / \mathrm{m}$, respectivamente, e foram obtidos empiricamente para representar a forma da encosta, enquanto os coeficientes $m$ e $n$ variam de acordo com as condições topográficas locais.

Para obter o fluxo acumulado e declividade foi utilizado o MDE derivado da imagem de radar ALOS/PALSAR, com resolução espacial de 12,5m, adquirido por meio do sistema de busca ASF (Alaska Satellite Facility). Inicialmente, realizou-se a preparação do MDE no software $\operatorname{ArcGIS}^{\circledR}$, que consistiu no recorte para a área em estudo (Clip), seguida pela aplicação da técnica de convolução bidimensional através da ferramenta Focal Statistics para remoção dos ruídos e correção das diferenças altimétricas entre pixels adjacentes, com posterior preenchimento das depressões (sinks) através da ferramenta Fill. O mapa de fluxo acumulado foi obtido com a ferramenta Flow Accumulation (Hydrology) e o mapa de declividade foi obtido com o uso da ferramenta Slope. 0 intervalo de classes de declividade foi definido com base nos critérios estabelecidos por Instituto Geológico (1993).

Os atributos do ITCTS, área de contribuição (As em $\mathrm{m}^{2} / \mathrm{m}$ ) e a tangente da declividade (Tan $\beta$ em radianos) foram calculados com base nesses mapas, através da calculadora de mapas (Map Algebra), utilizando as Equações 2 e 3. A Equação 2 foi utilizada para obter a área de contribuição específica, ou seja, área de drenagem por unidade de contorno e a Equação 3 para obter a tangente da inclinação. 
Onde: As é a área de contribuição específica $\left(\mathrm{m}^{2} / \mathrm{m}\right)$; fluxo acumulado representa o número de pixels que fluem para cada pixel descendente; Tan $\beta$ é a tangente da inclinação; e slope corresponde à declividade em radianos.

Para classificar o ITCTS adotou-se o método "Natural Breaks", que realiza um agrupamento dos valores maximizando as diferenças entre as classes. Desta forma as classes obtidas para a área de estudo foram: < 1.3 (baixo potencial erosivo); 1.3 a 3.1 (médio potencial erosivo) e 3.1 a 6.5 (alto potencial erosivo) e $>6.5$ (muito alto potencial erosivo).

\section{ÍNDICE DE VEGETAÇÃO POR DIFERENÇAS NORMALIZADAS}

O Índice de Vegetação por Diferenças Normalizadas (NDVI) é um dos indicadores numéricos mais amplamente utilizados para analisar imagens de sensoriamento remoto e avaliar a vegetação verde (Allison et al., 1989; Singh et al., 2004; Ayalew et al., 2020; Julien et al., 2011; Gandhi et al., 2015; Choubin et al., 2017).

Esse índice também foi utilizado significativamente para detectar mudanças na cobertura do solo causadas por atividades humanas, como construção, remoção de vegetação florestal, bem como analisar as mudanças da cobertura vegetal que ocasionam processos de degradação do meio físico, como os processos erosivos (Rocha et al., 2017; Baeza \& Paruelo, 2020; Spadoni et al., 2020).

A cobertura vegetal é um dos indicadores mais importantes para a erosão do solo e o NDVI nos permite delinear a distribuição da vegetação e do solo com base nos padrões característicos de refletância da vegetação verde (Karaburun, 2010).

Tucker (1979), usando as bandas de vermelho e do infravermelho próximo, introduziu o NDVI a partir dos dados de satélite MODIS. 0 valor NDVI varia entre $-1 \mathrm{e}+1$, onde os valores menores que zero indicam nuvens, neve e água e valores de -1 descrevem corpos de águas profundas, enquanto +1 indica vegetação densa.

0 NDVI está relacionado principalmente uma função do clima, terreno, vegetação/ecossistema e tipos de solo/hidrologia, o que pode ser calculado por meio da Equação 4.

$$
N D V I=\frac{N I R-R E D}{N I R+R E D}
$$

onde NIR = reflectância da vegetação na banda do infravermelho próximo e RED = reflectância da vegetação na banda do vermelho.

O NDVI para a área de estudo foi gerado a partir de imagens de satélite Landsat de 30 metros de resolução (bandas B3 - RED e B4 - NIR) dos anos de 2002, 2010 e 2018. As imagens estão disponíveis gratuitamente no banco de dados do Instituto Nacional de Pesquisas Espaciais (2021).

\section{PRODUÇÃO DE SEDIMENTOS}

A produção de sedimentos na área de estudo é monitorada por uma estação de qualidade da água gerenciada pela Agência Nacional de Águas (ANA) localizada no exutório da bacia (Figura 1). 0 monitoramento é realizado bimensalmente e conta com dados de 2003 a 2018.

A Formação Pirambóia, presente em $41 \%$ da área de estudo, é constituída por espessos corpos de arenitos de granulometria fina a média, com intercalações de argilitos e siltitos (Zaine, 1994). A presença de solos com textura arenosa em locais com relevo marcado pela presença de colinas (Planalto residual de São Carlos) favorece a ocorrência de processos erosivos e, consequentemente, o aporte de sedimentos nos canais de drenagem.

A identificação das erosões lineares (sulcos, ravinas e voçorocas) foi realizada através da interpretação visual das imagens de satélite disponíveis no software Google Earth Pro, considerando os anos de 2001, 2010 e 2018 Adotando o mesmo critério do Instituto de Pesquisas Tecnológicas (2012), foram utilizados alguns padrões visuais para orientar a identificação das erosões, como cabeceiras de drenagem, presença de vertentes com estradas e rodovias a montante, áreas com solo exposto. Posteriormente os pontos no formato KMZ foram 
inseridos no software ArcGIS e referenciados no sistema de coordenadas Universal Transverse Mercator (UTM) - Datum SIRGAS 2000 (Zona 23 S). A escala temporal adotada teve como base os outros mapas elaborados neste trabalho (NDVI e TCI), a fim de favorecer a análise conjunta dos dados.

\section{ANÁLISE DE DADOS}

A análise dos dados foi realizada em um ambiente de Sistema de Informações Geográficas (SIG), especificamente o software ArcGIS versão 10.5. Este software possui diversas funcionalidades e ferramentas, como ferramentas de hidrologia e ferramentas de processamentos de dados. Umas destas ferramentas é o tratamento de imagens de satélite, utilizada neste trabalho para elaboração dos fatores topográficos e do índice de vegetação por diferença normalizada (NDVI).

\section{RESULTADOS E DISCUSSÕES}

\section{Processos erosivos na Bacia Hidrográfica do Ribeirão do Roque}

Os processos erosivos existentes da área ocorrem predominantemente na região de ocorrência da Formação Pirambóia na bacia, como mostra a Figura 4. Analisando a distribuição espacial das erosões na área de estudo, observa-se que sua ocorrência está associada principalmente com a presença de solos arenosos, relevo com planaltos, uso do solo agrícola, e localizam-se principalmente próximo dos canais de drenagem. Para os três cenários analisados foram identificadas nas imagens de satélite do Google Earth, 58 processos erosivos em 2001, 39 em 2010 e 44 em 2018 (Figura 5).

Para compreender a susceptibilidade à erosão é fundamental considerar os fatores condicionantes como uso do solo, geologia e topografia. Além do mais, a adoção de práticas de controle fundamentada em princípios básicos de manejo do solo pode contribuir com o disciplinamento do escoamento superficial e assim evitar o surgimento de novas erosões, bem como controlar a sua evolução (Filizola et al., 2011).

Em 2001, tanto a presença de solo exposto como vegetação rasteira, favoreceram o surgimento de erosões lineares enquanto em 2010 e 2018, o cultivo agrícola (café) e a adoção de práticas conservacionistas (plantio em curva de nível) contribuíram para a redução do número de erosões. A Figura 6 mostra um exemplo de uma área na qual houve alteração do uso do solo com possível controle de erosão em 2010 e 2018.

Por outro lado, nota-se que algumas feições erosivas evoluíram de maneira significativa em termos de área e profundidade de 2001 para 2010, enquanto em 2018 apresentaram uma área relativamente menor. A Figura 7 exemplifica essa situação com base na imagem de satélite de 2018 e nas características do processo erosivo, na qual se observa uma possível adoção de prática de controle de processos erosivos e recuperação da paisagem. Observando a imagem de satélite de 2018, verifica-se que a feição foi parcialmente aterrada e as curvas de nível refeitas.

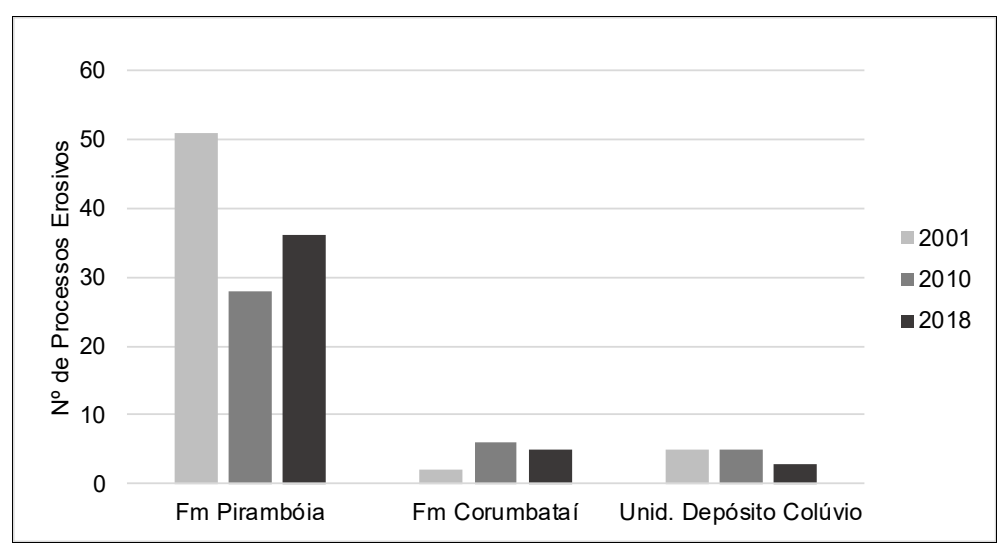

Figura 4 - Número de ocorrência de erosões lineares identificadas na área de estudo em função das unidades geológicas, nos anos de 2001, 2010 e 2018. Fonte: Autores (2020). 

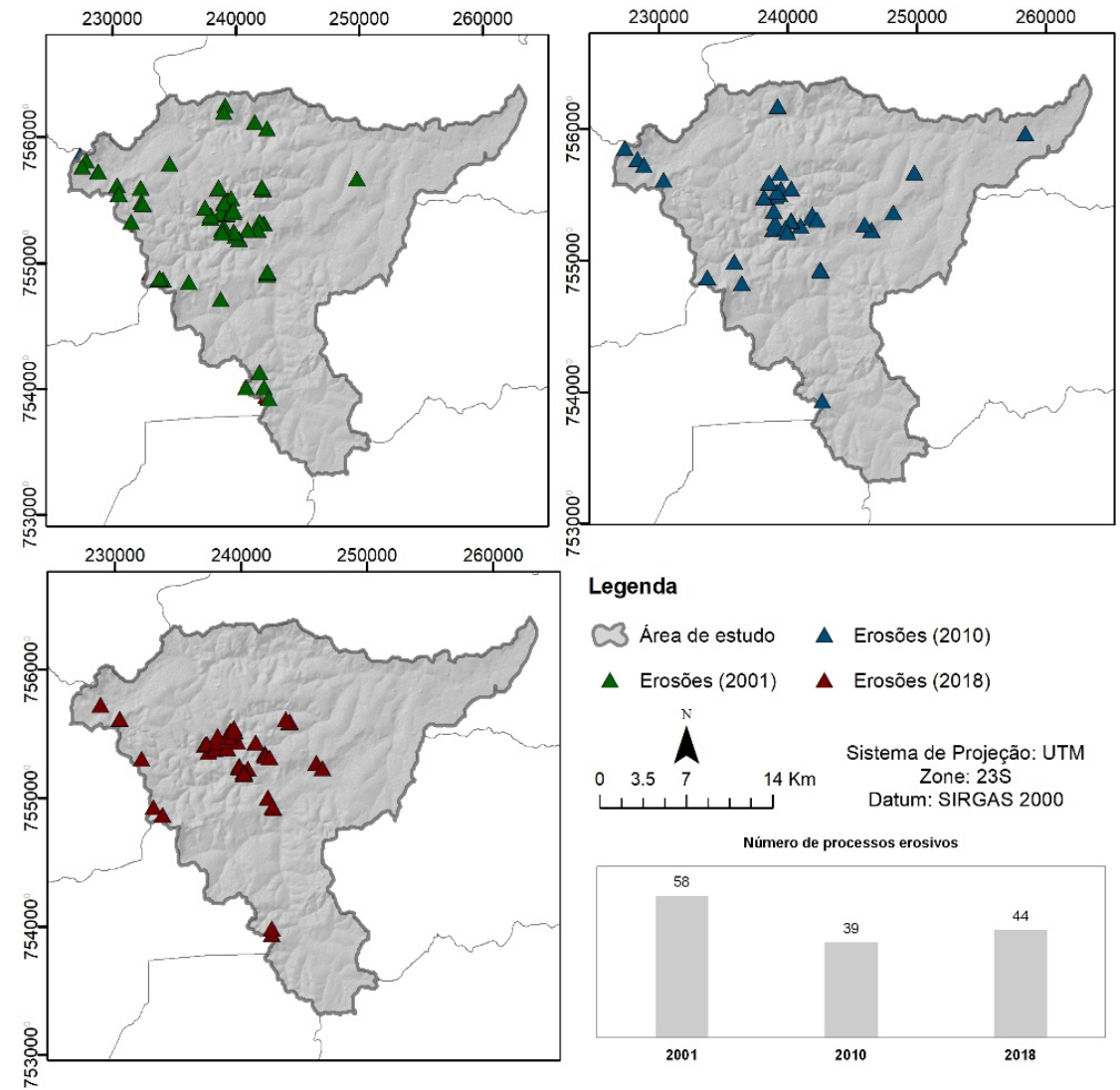

Figura 5 - Mapas de localização das erosões lineares identificadas neste estudo para os anos de 2001, 2010 e 2018. Fonte: Autores (2020).
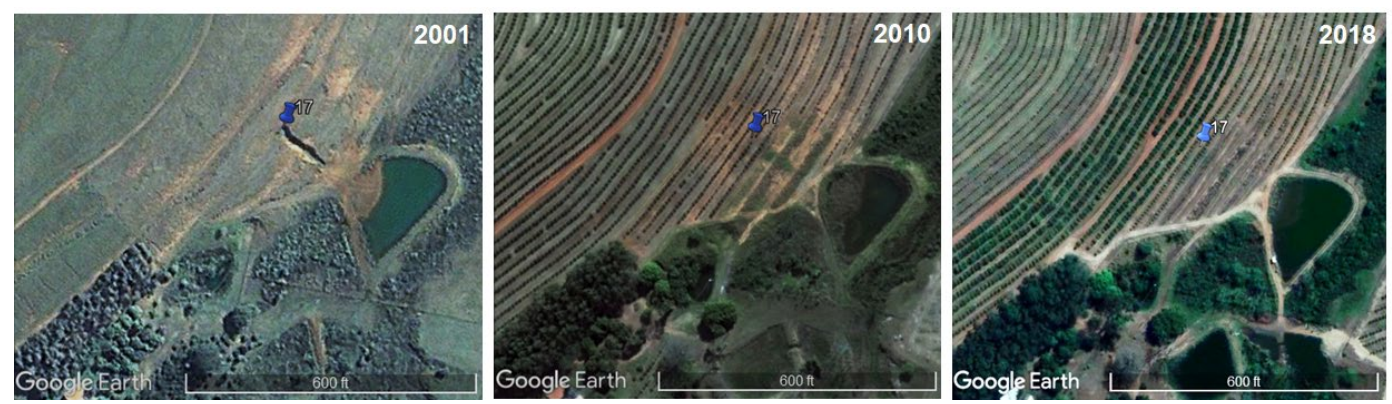

Figura 6 - Área com processo erosivo verificado em 2001, qual não está presente em 2010 e 2018. Fonte: Autores (2020).
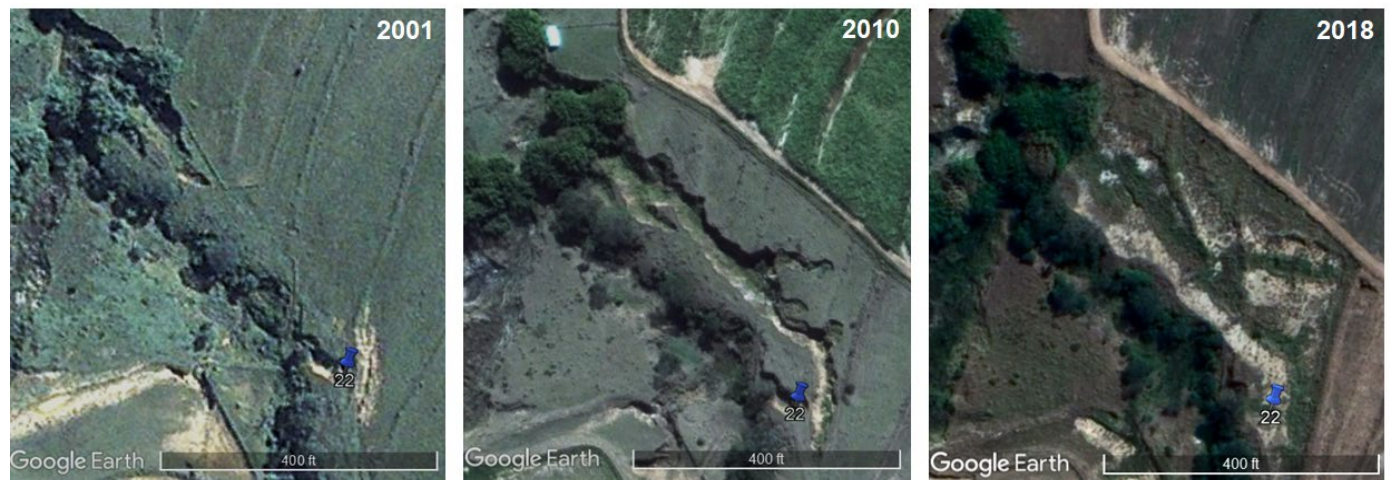

Figura 7 - Processo erosivo em 2001 e 2010, e possível adoção de prática de controle e recuperação em 2018, reduzindo a área erodida. Fonte: Autores (2020). 
É importante destacar que essas técnicas devem ser realizadas de maneira adequada, considerando princípios de manejo do solo e características do escoamento superficial, para evitar a reativação da erosão. Além do mais, é fundamental adotar medidas de manutenção para prolongar a vida útil das medidas de controle e recuperação (Filizola et al., 2011).

Conforme o mapa da Figura 8a a classe de declividade predominante na área em estudo corresponde ao intervalo de $5-10 \%(38,54 \%)$, seguida pelas classes $2-5 \%(22,24 \%)$ e $10-15 \%$ (18,39\%). Segundo o IG (1993), os terrenos com declividade entre 5 e $10 \%$ geralmente são inclinados, com relevo ondulado e escoamento superficial médio e rápido, e possuem poucos problemas de erosão superficial. A análise espacial mostrou que os processos erosivos existentes na área, ocorrem nos terrenos com declividade que variam de 15 a $20 \%$ e, segundo IG (1993), são áreas muito inclinadas com escoamento superficial rápido, com problemas de erosão superficial.

O mapa de fluxo acumulado representa a área de contribuição por unidade de contorno $\left(\mathrm{m}^{2} / \mathrm{m}\right)$, calculada considerando a contribuição do próprio pixel mais a contribuição dos pixels vizinhos. Desta maneira, é possível identificar as zonas com maior tendência de acumulação de água com base no ângulo da direção do fluxo. Pelo mapa de fluxo acumulado é possível identificar a influência das zonas de acumulação de água no surgimento de erosões, como mostra o detalhe B da Figura 8. As áreas com os maiores valores de acúmulo localizam-se nas porções mais baixas no relevo, em vales fechados com alta densidade de canais, enquanto as áreas com menores valores caracterizam-se pelos divisores topográficos da bacia.

A Figura 9 apresenta o mapa com o Índice Topográfico de Capacidade de Transporte de Sedimentos, no qual os valores para a área em estudo foram classificados em quatro classes (baixo $<1,3$; médio 1,3 a 3,1; alto 3,1 a 6,5; e muito alto potencial erosivo $>6,5$ ). Os locais com maiores valores indicam as áreas com maior potencial de energia do escoamento superficial e, portanto, maior capacidade de causar erosão ou proporcionar deposição de sedimentos.

Comparando o ITCTS com os pontos de erosão identificados nas imagens de satélite do Google Earth (2001, 2010 e 2018), verifica-se que, no geral, 8,5\% dos pontos mapeados ocorrem em áreas com baixo potencial erosivo, 56,7\% em áreas com médio potencial erosivo, $34 \%$ em áreas com alto potencial erosivo e $0,71 \%$ em áreas com muito alto potencial erosivo (Tabela 1 ). Ao analisar os três cenários (2001 a 2018) observa-se uma predominância dos pontos mapeados nas áreas com médio potencial erosivo, enquanto que para a classe "alto potencial erosivo" observa-se uma variação em relação à localização das erosões nessas áreas. As imagens de satélite das Figuras 6 e 7 mostram que a variação em relação à distribuição espacial dos pontos e o número de processos erosivos identificados podem estar relacionados a vários fatores como, por exemplo, alteração no uso do solo, manejo agrícola, e adoção de práticas de controle e recuperação.

Tabela 1 - Percentual de processos erosivos localizados nas áreas com baixo, médio e alto potencial erosivo.

\begin{tabular}{c|c|c|c|c}
\hline \multirow{2}{*}{ Classe ITCTS } & \multicolumn{4}{c}{ \% Processos erosivos nas classes de ICTS } \\
\cline { 3 - 5 } & & $\mathbf{2 0 0 1}$ & $\mathbf{2 0 1 0}$ & $\mathbf{2 0 1 8}$ \\
\hline Baixo potencial erosivo & $<1,3$ & 12,07 & 2,56 & 9,09 \\
\hline Médio potencial erosivo & 1,3 a 3,1 & 58,62 & 53,85 & 56,82 \\
\hline Alto potencial erosivo & 3,1 a 6,5 & 29,31 & 41,03 & 34,09 \\
\hline Muito alto potencial erosivo & $>6,5$ & 0 & 2,56 & 0 \\
\hline
\end{tabular}

Interpretando os resultados do ITCTS com base na classificação da declividade proposta pelo IG (1993), observa-se que as áreas que apresentam alto potencial erosivo correspondem a terrenos fortemente inclinados (declividade entre 15 e $25 \%$ ) com tendência de escoamento superficial rápido e problemas de erosão superficial, enquanto os locais com médio potencial erosivo caracterizam-se por terrenos inclinados (declividade entre 5 e 10\%) com escoamento superficial rápido, e presença de erosão em solos arenosos Comparando o mapa de ITCTS (Figura 9) com a distribuição espacial dos processos erosivos para os três cenários analisados (Figura 5) pode-se afirmar que o índice se mostrou eficiente, pois $90 \%$ dos pontos identificados nas imagens do Google Earth estão localizados em áreas classificadas com médio e alto potencial erosivo. 

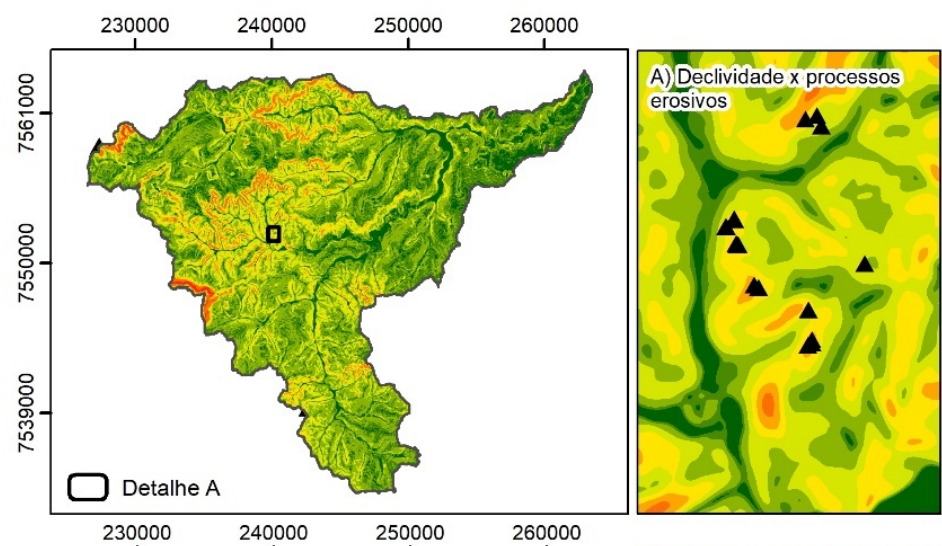

Legenda

3 Área de estudo

$\Delta$ Processos erosivos Slope $(\%)$

$3<2$

- 2 - 5

$5-10$

3 $10-15$

-3 $15-20$

C $20-25$

3 $25-50$

$3>50$
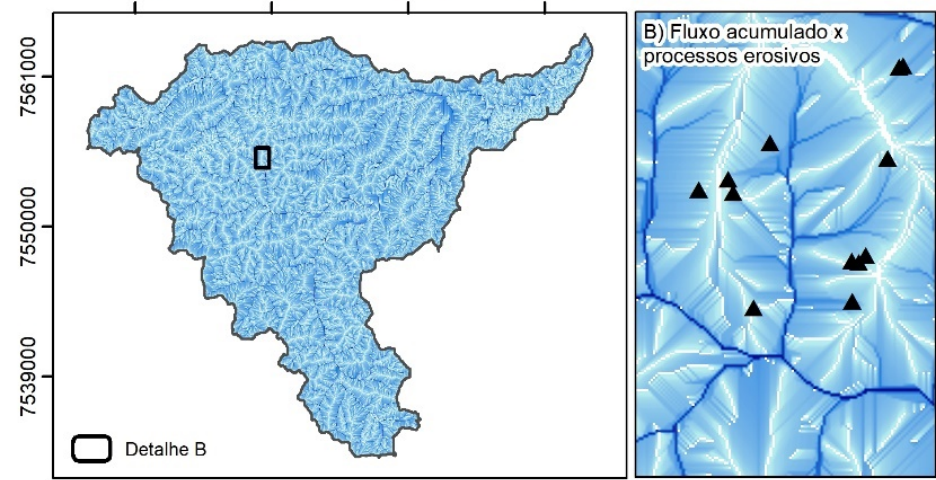

Fluxo acumulado

$\left(\mathrm{m}^{2} / \mathrm{m}-\log 10\right)$

Máx. : 6.49

Min. : 0

$02.755 .5 \quad 11 \mathrm{Km}$

Sistema de Projeção: UTM Zona: $23 S$

Datum: SIRGAS 2000

Figura 8 - Mapa de declividade (A); Mapa de fluxo acumulado (B). Fonte: Autores (2020).

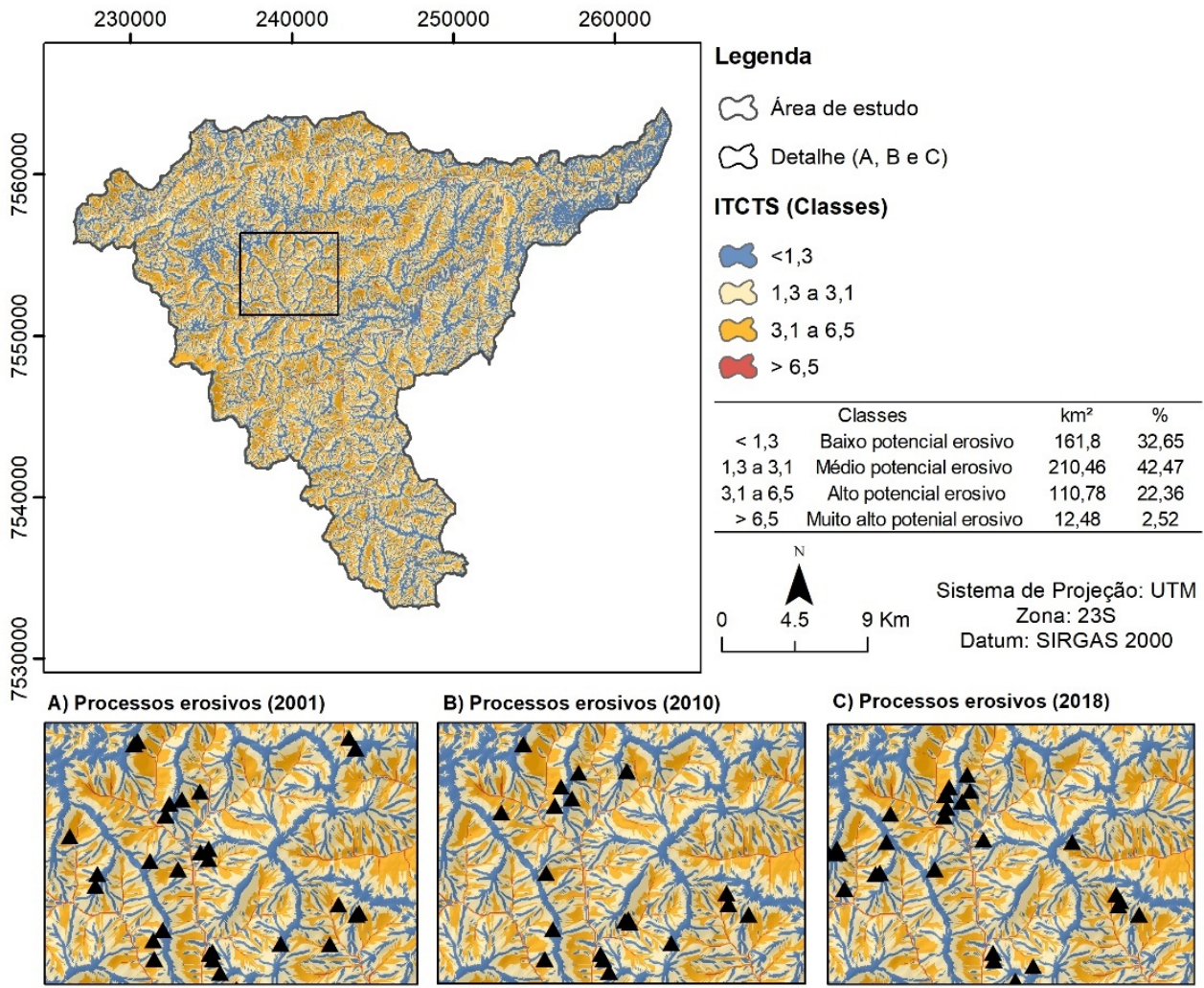

Figura 9 - Mapa do Índice topográfico de capacidade de transporte de sedimentos (ITCTS) e detalhe das regiões com erosões identificadas em 2001 (A), 2010 (B) e 2018 (C). Fonte: Autores (2020). 


\section{NDVI E PRODUÇÃO DE SEDIMENTOS}

Os resultados mostram que a variação temporal do NDVI comprova o fato de que, com o passar dos anos, a vegetação foi reduzida na área de estudo e substituída por outros tipos de uso e ocupação do solo. A área central da bacia hidrográfica é onde se encontram a maior parte dos processos erosivos da área de estudo (Figura 5) e nesta mesma área podemos observar na Figura 10 a remoção de vegetação nativa em detrimento de outros tipos de cultura, o que possivelmente potencializou a ocorrência de erosão.

O NDVI para o ano de 2002 (Figura 10) indica que a maioria da área de estudo encontra-se na categoria de moderada vegetação (NDVI entre 0,4 a 0,6), enquanto que para o ano de 2018 a maior parte da área de estudo encontrava-se nas categorias de baixa e muito baixa vegetação, ou seja, de 0 a 0,4 (Figura 11). Os valores acumulados do NDVI apresentados no gráfico na Figura 12 comprovam este fato e mostram a redução da cobertura vegetal em 2018 entre as classes com NDVI muito baixo (entre 0 e 0,2) e baixo $(0,2$ a 0,4) em comparação com os anos de 2002 e 2010.

Entre 2010 e 2018 o número de processos erosivos ativos na área em estudo aumentou e, considerando os valores do NDVI, acredita-se que a ausência de cobertura vegetal pode ter sido um dos fatores responsáveis pelo surgimento de novos processos.

A Figura 12 mostra a produção de sedimentos (mg/L) para a estação de monitoramento localizada no exutório da bacia hidrográfica. Entre os anos de 2008 e 2011 percebe-se um aumento significativo nas concentrações de sedimentos nos cursos d'água, que pode ser resultado da alteração do uso do solo na área de estudo (majoritariamente o aumento da produção agrícola). Alkimim et al., (2015) e Strassburg et al., (2017) destacam o crescimento da agricultura no estado de São Paulo nas últimas décadas, com a intensificação do uso da terra e a substituição da vegetação natural por cultivo agrícola, como cana-de-açúcar.
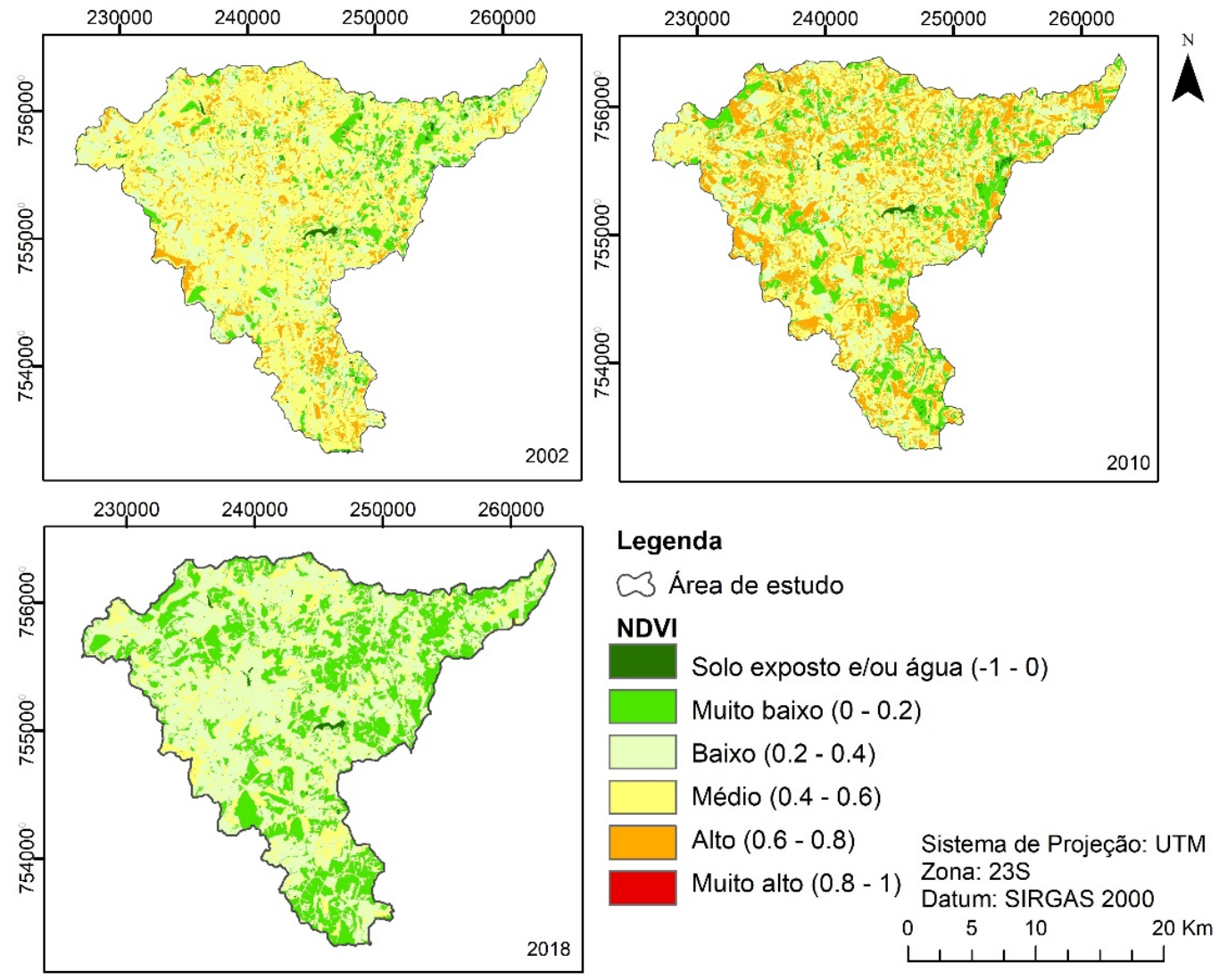

Figura 10 - Mapa de Índice de Vegetação por Diferenças Normalizadas (NDVI) para os anos de 2000, 2010 e 2018. Fonte: Autores (2020). 


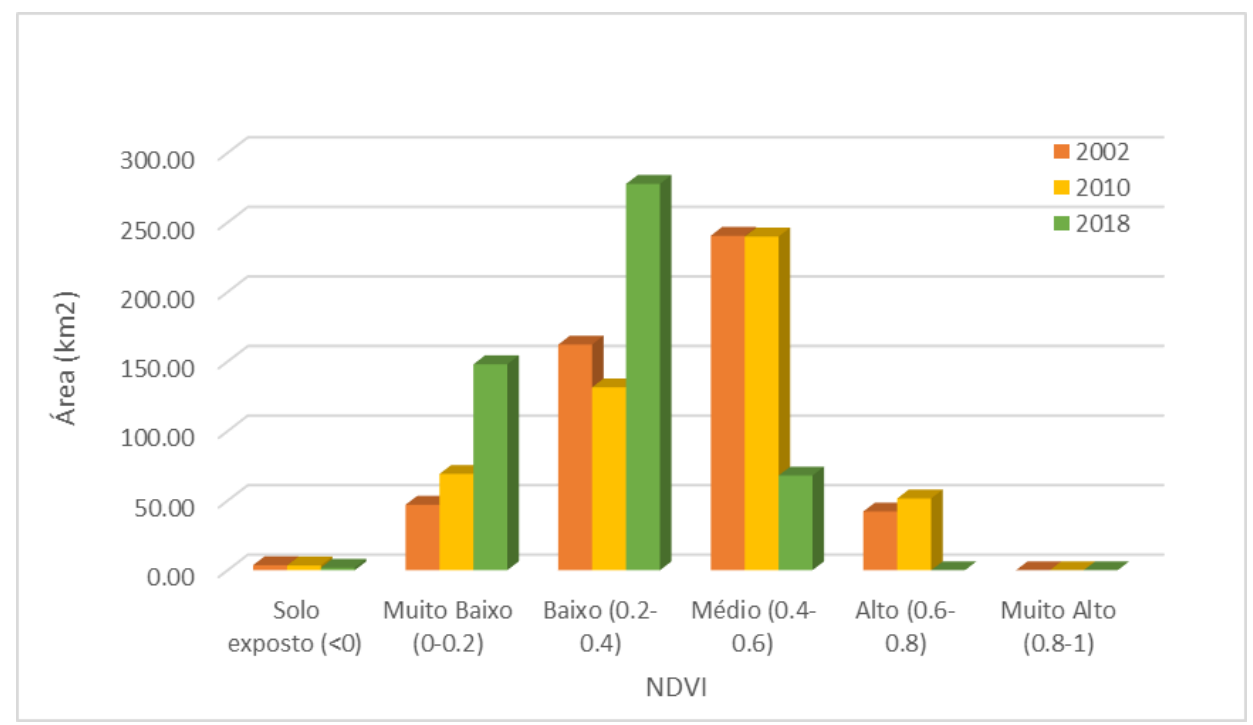

Figura 11 - Ocorrências das classes de NDVI em km². Fonte: Autores (2020).

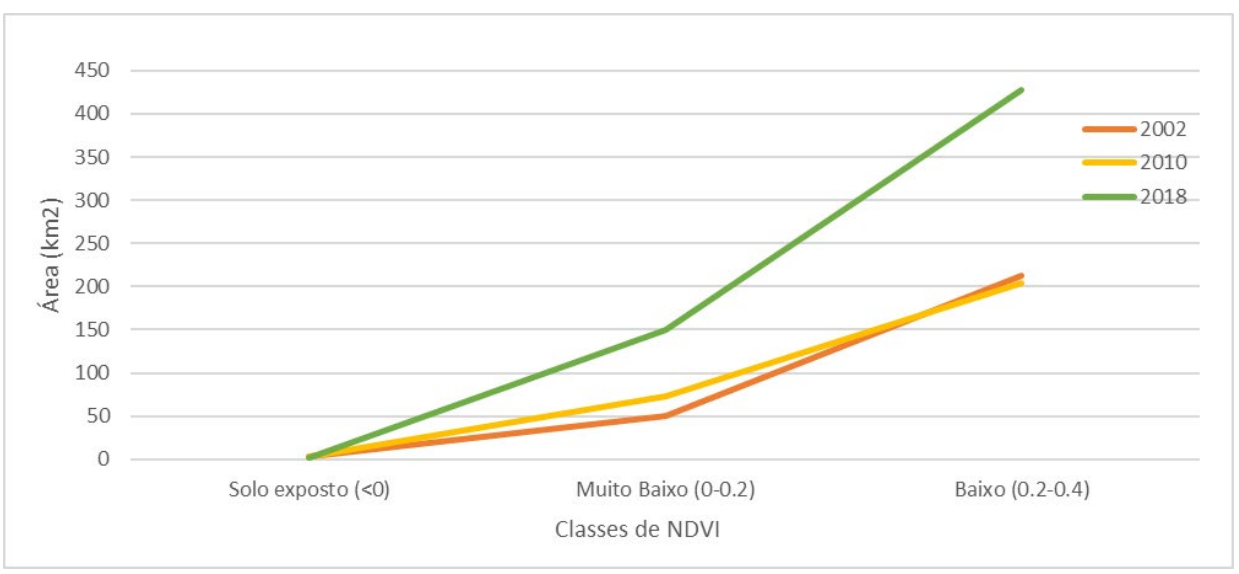

Figura 12 - Valores acumulados de NDVI. Fonte: Autores (2020).

O ITCTS tem como vantagem a fácil obtenção dos atributos primários a partir do MDE que por sua vez, podem ser manipulados em softwares open source e assim permitem interpretar o efeito na topografia na erosão do solo. Desta maneira, constitui uma importante ferramenta no âmbito do planejamento de bacias, pois possibilita selecionar áreas prioritárias para implantação de medidas de conservação do solo e da água, recuperação ambiental e restrição de uso. É importante destacar que uma análise mais robusta sobre as áreas potenciais só é eficaz se outros fatores condicionantes, como geologia, solos, vegetação e clima.

Analisando os resultados obtidos neste trabalho, verifica-se que as áreas com maior potencial de ocorrência de erosão (conforme o índice de capacidade de transporte de sedimento da Figura 9) estão localizadas em terrenos mais íngremes, próximo das cabeceiras de drenagem, e mostraram conformidade com os pontos identificados nas imagens de satélite disponibilizadas pelo Google Earth.

Nos três cenários analisados, a maioria dos pontos mapeados estava localizada em áreas com médio potencial erosivo (> 50\%), enquanto para a classe "alto potencial erosivo" observou-se uma variação de 29 a $41 \%$ ao longo dos cenários. Nesse caso, as características do relevo têm maior influência na ocorrência de processos erosivos, especialmente nos terrenos com maior declividade, que condicionam fluxo com maior energia de transporte e favorecem o surgimento de erosões. Além disso, é importante destacar que as áreas com médio e alto potencial erosivo ocorrem na região geológica da Formação Pirambóia, que por sua vez também atua como um dos principais fatores condicionantes da erosão, devido à presença de solos arenosos altamente susceptíveis a erosão. 


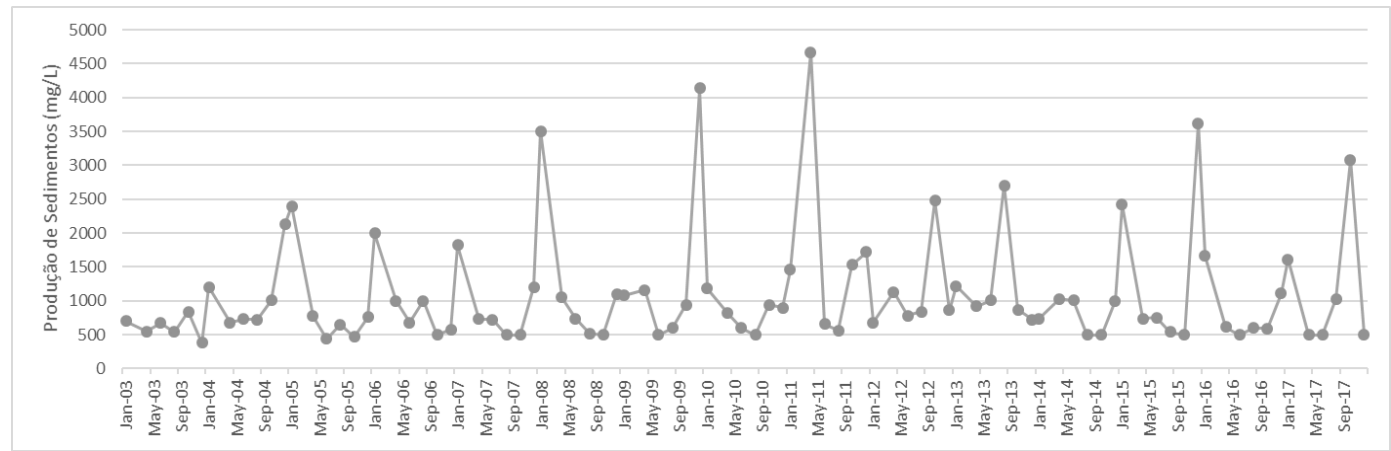

Figura 13 - Produção de sedimentos na área de estudo. Fonte: Autores (2020).

As alterações antrópicas que ocorreram ao longo dos anos, analisadas com base no NDVI mostram que a baixa cobertura vegetal observada principalmente em 2018 pode ter contribuído com o aumento de erosões na área em estudo. Conforme o mapa da Figura 10 as regiões classificadas como baixa $(0,2-0,4)$ e muito baixa $(0-0,2)$ cobertura vegetal estão localizadas nas áreas com potencial de ocorrência de erosão (ITCTS).

Os resultados da produção dos sedimentos (Figura 13) mostram que durante os períodos úmidos (outubro a março) ocorrem os picos de produção de sedimentos. Estes sedimentos que são transportados para os cursos d'água podem ser advindos das ravinas e voçorocas (identificadas a partir das imagens de satélite) e também com a produção agrícola intensa, conforme apresentado nos estudos de Alkimim et al., (2015) e Strassburg et al., (2017).

Considerando que a área em estudo apresenta potencial de ocorrência a erosão, recomenda-se a adoção de práticas agrícolas de conservação do solo e da água, para evitar o surgimento de novas feições, bem como a evolução para processos erosivos mais complexos, como voçorocas. Tais medidas são fundamentais, pois as erosões lineares podem ocasionar processos de degradação diversos na área.

\section{CONCLUSÕES}

0 índice topográfico de capacidade de transporte de sedimento (ITCTS) auxiliou na identificação espacial das áreas com maior potencial de ocorrência de erosão e deposição na área em estudo.

O NDVI permitiu analisar as alterações que ocorreram na vegetação e sua influência na ocorrência dos processos erosivos. Os resultados indicaram aumento da produção de sedimentos entre os anos de 2008 e 2011, o que deve estar relacionado às mudanças do uso e ocupação do solo decorrente da agricultura e consequentes processos erosivos.

Os resultados obtidos indicam que a relação entre valores de NDVI, registros de processos erosivos e produção de sedimentos fornece uma perspectiva de impactos ocasionados na bacia, em termos de degradação do solo e sua influência na degradação dos mananciais superficiais, podendo comprometer ecossistemas e saúde pública.

Tal combinação de processos de análise pode dar suporte a propostas de gestão e gerenciamento para a bacia, bem como outras tantas na região com condições semelhantes de solo e de uso e cobertura.

\section{AGRADECIMENTOS}

Os autores informam que o presente trabalho foi realizado com apoio da Coordenação de Aperfeiçoamento de Pessoal de Nível Superior - Brasil (CAPES) - Código de Financiamento 001. Os autores agradecem ao Conselho Nacional de Desenvolvimento Científico e Tecnológico (CNPq), pelo apoio financeiro (Processos 428428/2018-5 e 306074/2018-4).

\section{REFERÊNCIAS}

Alkimim, A., Sparovek, G., \& Clarke, K. C. (2015). Converting Brazil's pastures to cropland: an alternative way to meet sugarcane demand and to spare forestlands. Applied Geography, 62(1), 75-84.

Allison, E. W., Brown, R. J., Press, H. E., \& Gairns, J. G. (1989). Monitoring drought affected vegetation with AVHRR. In 12th Canadian Symposium on Remote Sensing Geoscience and Remote Sensing Symposium (pp. 1965-1967). IEEE. 
Alvares, C. A., Stape, J. L., Sentelhas, P. C., Gonçalves, J. L. M., \& Sparovek, G. (2013). Köppen’s climate classification map for Brazil. Meteorologische Zeitschrift, 22(6), 711-728.

Anjinho, P. S., Campos, L. G., Mauad, F. F., \& Moschini, L. E. (2018). Geotecnologias aplicadas à análise do riso a contaminação dos aquíferos da Bacia Hidrográfica do rio São Roque - SP. Águas Subterrâneas, 32(3), 1-11.

Asselman, N. E. M., Middelkoop, H., Van Dijk, P. M. (2003). The impact of changes in climate and land use on soil erosion, transport and deposition of suspended sediment in the River Rhine. Hydrological Processes, 17, 3225-3244.

Ayalew, D. A., Deumlich, D., Šarapatka, B., \& Doktor, D. (2020). Quantifying the sensitivity of NDVI-based C factor estimation and potential soil erosion prediction using Spaceborne earth observation data. Remote Sensing, 12(7), 1136.

Baeza, S., \& Paruelo, J. M. (2020). Land use/land cover change (2000-2014) in the Rio de la Plata grasslands: an analysis based on MODIS NDVI time series. Remote Sensing, 12(3), 381.

Carvalho, D. F. D., Durigon, V. L., Antunes, M. A. H., Almeida, W. S. D., \& Oliveira, P. T. S. D. (2014). Predicting soil erosion using Rusle and NDVI time series from TM Landsat 5. Pesquisa Agropecuária Brasileira, 49(3), 215-224.

Choubin, B., Solaimani, K., Roshan, M. H., \& Malekian, A. (2017). Watershed classification by remote sensing indices: a fuzzy c-means clustering approach. Journal of Mountain Science, 14(10), 2053-2063.

Filizola, H. F., Almeida Filho, G. S., Canil, K., Souza, M. D., \& Gomes, M. A. F. (2011). Controle dos processos erosivos lineares (ravinas e voçorocas) em áreas de solos arenosos (Circular Técnica, No. 22). São Paulo: EMBRAPA.

Gandhi, G. M., Parthiban, S., Thummalu, N., \& Christy, A. (2015). NDVI: vegetation change detection using remote sensing and gis: a case study of Vellore District. Procedia Computer Science, 57, 1199-1210.

Guerra, A. J. T. (1998). Processos erosivos nas encostas. In A. J. T. Guerra \& S. B. Cunha (Eds.), Geomorfologia: uma atualização de bases e conceitos (pp. 149-199). Rio de Janeiro: Bertrand Brasil.

Instituto de Pesquisas Tecnológicas - IPT. (2012). Relatório técnico: IPT/DAEE. São Paulo: IPT.

Instituto Geológico - IG. (1993). Subsídios do meio físico-geológico ao planejamento do município de Campinas (SP). Relatório de Integração (Vol. I, 85 p.). São Paulo: IG.

Instituto Geológico - IG. (2017). Unidades básicas de compartimentação do meio físico do Estado de São Paulo. São Paulo: IG.

Instituto Nacional de Pesquisas Espaciais - INPE. (2018). Imagens de satélites. Recuperado em 19 de maio de 2021, de http://www.dgi.inpe.br/documentacao/satelites

Instituto Nacional de Pesquisas Espaciais - INPE. (2021). Recuperado em 19 de maio de 2021, de http://www.dgi.inpe.br/CDSR/

Japan Aerospace Exploration Agency - JAXA. (2019). Calibration result of ALOS-2/PALSAR-2 JAXA standard products. Tokyo.

Jin, K., Cornelis, W. M., Gabriels, D., Schiettecatte, W., De Neve, S., Lu, J., Buysse, T., Wu, H., Cai, D., Jin, J., \& Harman, R. (2008). Soil management effects on runoff and soil loss from field rainfall simulation. Catena, 75, 191-199.

Julien, Y., Sobrino, J. A., Mattar, C., Ruescas, A. B., Jiménez-Muñoz, J. C., Sòria, G., Hidalgo, V., Atitar, M., Franch, B., \& Cuenca, J. (2011). Temporal analysis of normalized difference vegetation index (NDVI) and land surface temperature (LST) parameters to detect changes in the Iberian land cover between 1981 and 2001. International Journal of Remote Sensing, 32(7), 2057-2068.

Karaburun, A. (2010). Estimation of C factor for soil erosion modeling using NDVI in Buyukcekmece watershed. Ozean Journal of Applied Sciences, 3(1), 77-85.

Minella, J. P. G., \& Merten, G. H. (2012). Índices topográficos aplicados à modelagem agrícola e ambiental. Ciência Rural, 42(9), 1575-1582.

Moore, I. D., \& Burch, G. J. (1986). Physical basis of the length-slope factor in the universal soil loss equation 1. Soil Science Society of America Journal, 50(5), 1294-1298.

Ouyang, W., Hao, F., Skidmore, A. K., \& Toxopeus, A. G. (2010). Soil erosion and sediment yield and their relationships with vegetation cover in upper stream of the Yellow River. The Science of the Total Environment, 409(2), 396-403.

Rocha, N. A., De Sena, Í. S., Casagrande, P. B., De Castro, M. M., Fonseca, B. M., \& Moura, A. C. M. (2017). Studies of volumetric relation between vegetation and buildings using LIDAR data and NDVI to propose urban parameters. Revista Brasileira de Cartografia, 69(8), 1456-1475. 
Ross, J. L., \& Moroz, I. C. (1997). Mapa geomorfológico do Estado de São Paulo. São Paulo: FFLCHUSP/IPT/FAPESP.

Singer, M. J., \& Shainberg, I. (2004). Mineral soil surface crusts and wind and water erosion. Earth Surface Processes and Landforms, 29, 1065-1075.

Singh, D., Herlin, I., Berroir, J. P., Silva, E. F., \& Meirelles, M. S. (2004). An approach to correlate NDVI with soil colour for erosion process using NOAA/AVHRR data. Advances in Space Research, 33(3), 328-332.

Spadoni, G. L., Cavalli, A., Congedo, L., \& Munafò, M. (2020). Analysis of Normalized Difference Vegetation Index (NDVI) multi-temporal series for the production of forest cartography. Remote Sensing Applications: Society and Environment, 20, 100419.

Strassburg, B. B. N., Brooks, T., Feltran-Barbieri, R., Iribarrem, A., Crouzeilles, R., Loyola, R., Latawiec, A. E., Oliveira Filho, F. J. B., Scaramuzza, C. A. M., Scarano, F. R., Soares-Filho, B., \& Balmford, A. (2017). Moment of truth for the Cerrado hotspot. Nature Ecology \& Evolution, 1(4), 1-2.

Tucker, C. J. (1979). Red and photographic infrared linear combinations for monitoring vegetation. Remote Sensing of Environment, 8(2), 127-150.

Vaz, A. P. M. S., Ramos, S. M., \& Froehner, S. J. (2021). Bacia hidrográfica do rio balsas: diagnóstico físico e avaliação qualitativa de áreas suscetíveis à erosão. Engenharia Sanitaria e Ambiental, 26(1), 77-87.

Vrieling, A., de Jong, S. M., Sterk, G., \& Rodrigues, S. C. (2008). Timing of erosion and satellite data: a multiresolution approach to soil erosion risk mapping. International Journal of Applied Earth Observation and Geoinformation, 10(3), 267-281.

Wilson, J. P., \& Gallant, J. C. (1996). EROS: A grid-based program for estimating spatially-distributed erosion indices. Computers \& Geosciences, 22(7), 707-712.

Wischmeier, W. H., \& Smith, R. E. (1978). Predicting rainfall erosion losses: a guide to conservation planning. United States Department of Agriculture - USDA, Agriculture handbook. Washington: U.S. Government Printing Office.

Zaine, J. E. (1994). Geologia da formação Rio Claro na Folha Rio Claro (SP) (Dissertação de mestrado). Instituto de Geociências e Ciências Exatas, Universidade Estadual Paulista, Rio Claro.

\section{Contribuições dos autores:}

Monique de Paula Neves: levantamento de dados, análise dos dados e redação do artigo.

Franciane Mendonça dos Santos: levantamento de dados, análise dos dados e redação do artigo.

José Augusto di Lollo: análise e discussão dos resultados. 